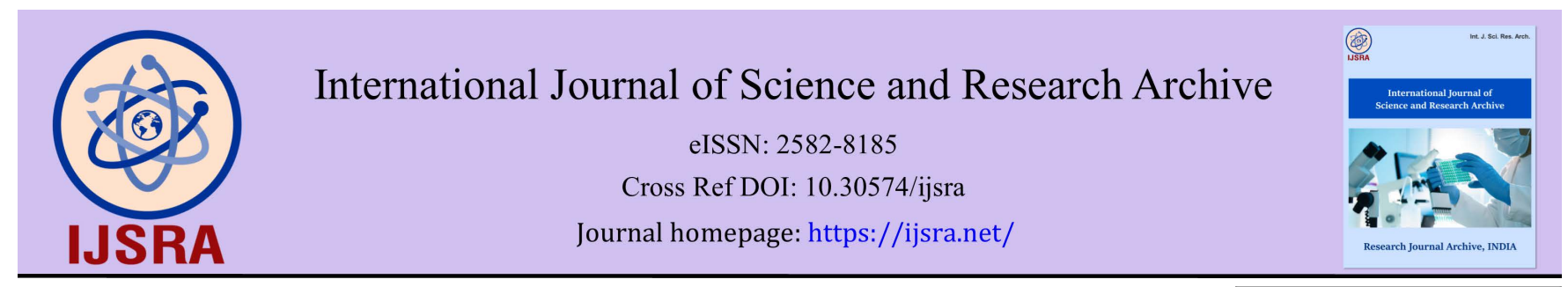

(RESEARCh ARTiCle)

Check for updates

\title{
The effect of integrative rehabilitation treatment on upper extremity ability of patients with central nervous system injury
}

Jung-Ho Lee*

Department of Physical Therapy, Kyungdong University: 815, Gyeonhwon-ro, Munmak-eup, Wonju-si, Gangwon-do, Republic of Korea.

International Journal of Science and Research Archive, 2021, 03(01), 071-078

Publication history: Received on 24 June 2021; revised on 27 July 2021; accepted on 29 July 2021

Article DOI: https://doi.org/10.30574/ijsra.2021.3.1.0109

\begin{abstract}
Purpose: The aim of this study was to test and develop a rehabilitation treatment protocol that maximizes the improvement of upper limb. In addition, this study was conducted to present a basic protocol for an integrative rehabilitation treatment method.

Methods: Patients diagnosed with stroke and exhibiting movement disorders in paralyzed limbs were randomly assigned to an experimental group and a control group. The experimental group received proprioceptive neuromuscular facilitation, extracorporeal shock wave therapy, and taping therapy. The control group received general exercise therapy and functional electrical stimulation. The treatment program for each group was conducted two times a week for four weeks, and exercise was performed for 60 minutes per session.
\end{abstract}

Results: There were statistically significant differences in the grip power with muscular strength of the shoulder joint flexor, extensor, adductor and abductor muscles before and after treatment in the experimental group and control group.

Conclusion: In conclusion, in order to improve upper extremity ability, a treatment method that can be applied at the same time and a method for managing the cause of the patient's limitations must be applied together.

Keywords: Muscle; Nerve; Ability; Rehabilitation

\section{Introduction}

In daily life, the upper limbs frequently perform complicated motions, such as functional reaching out, grasping, and lifting [1]. The reaching out motion, for example, is the result of the complex coordination of large movements and delicate movements of various joints. The organic control of the nervous system and motor system is essential for proper activities in daily life [2].

Stroke is a syndrome caused by blood circulation disorders in the brain, such as cerebral hemorrhage and cerebral infarction [3]. Strokes are accompanied by physical disability, a decline in cognitive functions, and mental disorders, often causing a decline in the quality of life. After a stroke, one-third die, one-third experience permanent disability, and the other one-third have temporary disability [4].

\footnotetext{
${ }^{*}$ Corresponding author: Jung-Ho Lee

Department of Physical Therapy, Kyungdong University: 815, Gyeonhwon-ro, Munmak-eup, Wonju-si, Gangwon-do, Republic of Korea.
}

Copyright (@ 2021 Author(s) retain the copyright of this article. This article is published under the terms of the Creative Commons Attribution Liscense 4.0. 
The upper limb muscle weakening, spasticity, imbalance, catatonia, and dysesthesia found in stroke patients bring about a loss of upper limb motor control and cause many limitations in independent daily living activities, such as eating, dressing, and personal care. After a stroke, as described above, many disorders appear in patients, and if they do not receive proper treatment in the early stages, they experience a lot of secondary limitations $[5,6]$.

The proprioceptive senses detect the postures of the limbs in stationary states and movements in dynamic states, and recognize the movements of muscles, the postures of the joints, and the object in contact [7].

Proprioceptive neuromuscular facilitation is an exercise therapy that improves function by stimulating proprioceptors for the promotion and suppression of specific muscle groups and is applied to stroke patients to restore and strengthen normal movement patterns, postural responses, and walking ability [8].

Kinesio taping is divided into muscle taping and corrective taping methods; they differ in the direction in which the force is applied to the tape and the tape elasticity. The advantage of taping is that it can be applied in parallel with other treatments without causing discomfort to the patient [9].

Therefore, the aim of this study was to develop a rehabilitation treatment protocol that maximizes the improvement of upper limb activity in stroke patients. In addition, this study was conducted to present a basic protocol for an integrative rehabilitation treatment method.

\section{Material and methods}

\subsection{Subjects}

In this study, 31 patients who were diagnosed with stroke and exhibited movement disorders in their paretic limbs were randomly assigned to an experimental group of 16 persons and a control group of 15 persons (Figure 1). The experimental group received extracorporeal shock wave therapy and then proprioceptive neuromuscular facilitation treatment with taping applied. The control group received general physical therapy.

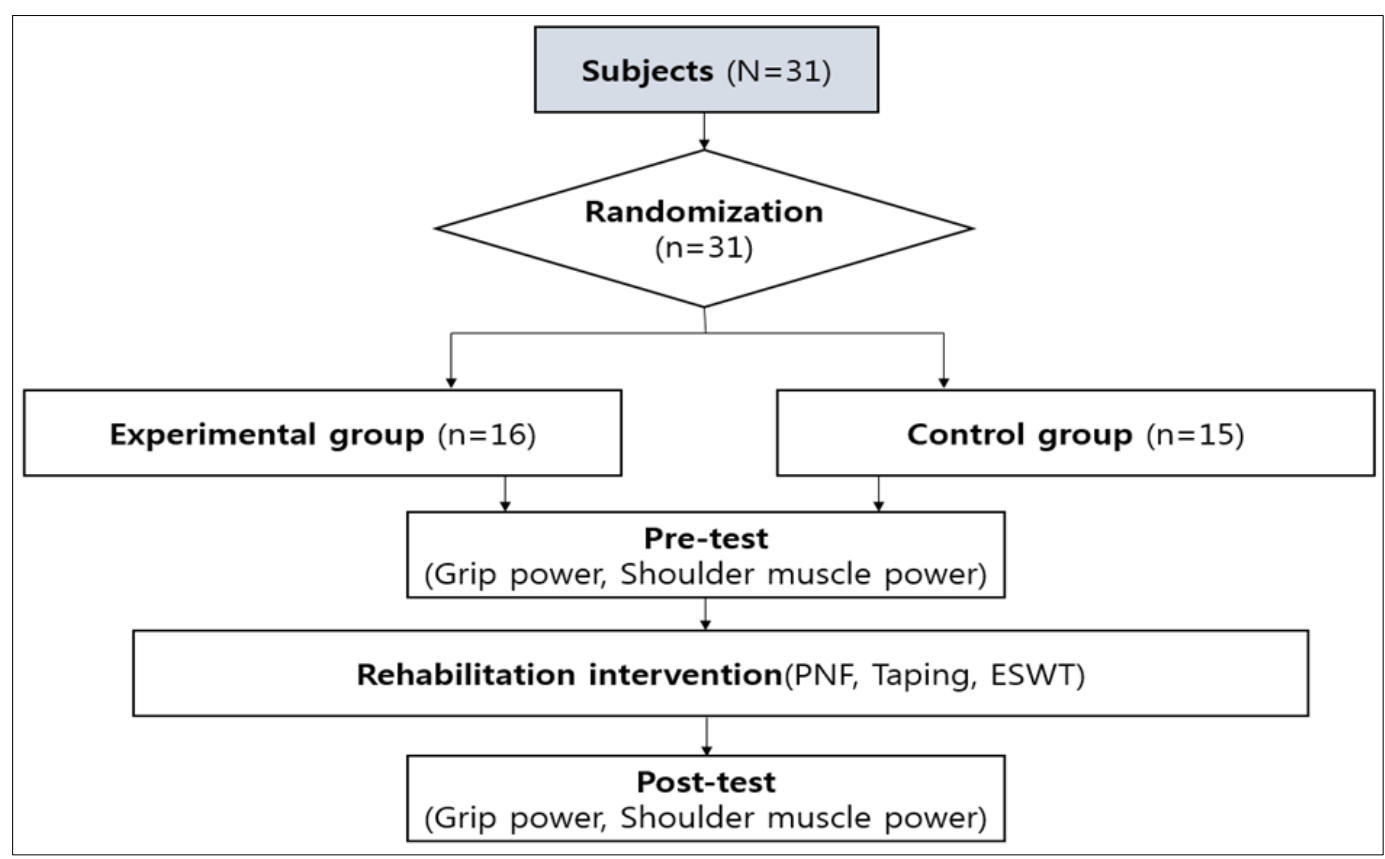

Figure 1 Flow chart

\subsection{Study design}

All study subjects agreed to the experiment after hearing detailed explanations about the contents of the study in advance. Those that agree to enter the study signed a written informed consent. All experimental procedures were carried out based on the Declaration of Helsinki. 


\subsection{Inclusion criteria}

Inclusion criteria were set as those

- Subjects had consented to the treatment program

- Subjects had no cognitive impairment, with a score on the Mini-Mental State Examination not lower than 24 points

- Subjects had no orthopedic problem, such as fractures or peripheral nerve damage in the upper limbs

- Subjects had no problems with vision or hearing

- Subjects had no subluxation of the shoulder joint on the paretic side.

\subsection{Exclusion criteria}

Exclusion criteria were set as those

- Subjects had heart failure

- Subjects had mental disorders or were taking psychoactive drugs

- Subjects had vomiting or dizziness

- Subjects had any cerebellum-related disease.

\section{Evaluation method}

\subsection{Grip power}

Grip strength was measured with a digital dynamometer. Before measuring grip strength, the dynamometer and measurement procedure were explained so that the subjects could understand them.

The grip strength was measured three times using the dominant hand, and the subjects were instructed to rest for $60 \mathrm{~s}$ after each measurement.

\subsection{Shoulder joint muscle power}

To measure shoulder joint strength, the strength of the muscles around the paralyzed shoulder joint was measured. The strength of the flexor and adductor muscles was measured using a portable strength dynamometer (PowerTrack II, JTech, USA).

\section{Treatment methods}

\subsection{Proprioceptive neuromuscular facilitation}

In order to apply the treatment of proprioceptive neuromuscular facilitation, the pattern treatment used in proprioceptive neuromuscular facilitation was used.

In proprioceptive neuromuscular facilitation, there are various pattern treatments to enhance muscle strength. In this study, a combined upper and lower limb pattern was used to strengthen the patient's muscle strength. To prevent muscle fatigue in the subjects, one-minute breaks were scheduled after every 10 repetitions.

In this study, upper (Table 1,2) and lower (Table 3,4) extremity patterns were used as shown in the table below.

\subsection{Taping}

In this study, Kinesio taping was used to apply taping therapy. Kinesio taping is a taping that has elasticity and is known to support muscle movement and increase muscle activity. The taping was applied to the muscles around the shoulder joint, and the applied muscles are deltoid, supraspinous, and infraspinous, which play an important role in the movement of the shoulder joint. 
Table 1 PNF upper limb D1 pattern used for rehabilitation intervention

\begin{tabular}{|l|c|c|c|}
\hline \multicolumn{2}{|c|}{ D1 flexion Pattern } & \multicolumn{2}{c|}{ D1 extension pattern } \\
\hline Shoulder & Flexion, External rotation, Adduction & Shoulder & Extension, Internal rotation, Abduction \\
\hline Forearm & Supination & Forearm & Pronation \\
\hline Wrist & Flexion, Radial deviation & Wrist & Extension, Ulnar deviation \\
\hline Finger & Flexion & Finger & Extension \\
\hline
\end{tabular}

Table 2 PNF upper limb D2 pattern used for rehabilitation intervention

\begin{tabular}{|l|c|l|c|}
\hline \multicolumn{2}{|c|}{ D2 flexion Pattern } & \multicolumn{2}{c|}{ D2 extension pattern } \\
\hline Shoulder & Flexion, External rotation, Abduction & Shoulder & Extension, Internal rotation, Adduction \\
\hline Forearm & Supination & Forearm & Pronation \\
\hline Wrist & Extension, Radial deviation & Wrist & Flexion, Ulnar deviation \\
\hline Finger & Extension & Finger & Flexion \\
\hline
\end{tabular}

\subsection{Extracorporeal shock wave therapy}

In this study, a shock wave generator was used for extracorporeal shock wave therapy. The same shock wave stimulation was applied to individual sites. The extracorporeal shock wave therapy device was applied in a range where the patient did not feel pain, and was used at the patient's muscle-tendon junction according to the prescribed manual.

\section{Statistical analysis method}

For data analysis, SPSS 18.0 was used in this study. All data were expressed as mean and standard deviation. In this study, a corresponding t-test was performed to compare the pre-test and post-test measured values of the two groups. In addition, an independent t-test was used to test the difference in treatment effect between groups.

Table 3 PNF lower limb D1 pattern used for rehabilitation intervention

\begin{tabular}{|c|c|c|c|}
\hline \multicolumn{2}{|l|}{ D1 flexion Pattern } & \multicolumn{2}{|c|}{ D1 extension pattern } \\
\hline Hip & Flexion, External rotation, Adduction & Hip & Extension, Internal rotation, Abduction \\
\hline Foot & Dorsal flexion, Inversion & Foot & Plantar flexion, Eversion \\
\hline Toes & Extension & Toes & Flexion \\
\hline
\end{tabular}

Table 4 PNF lower limb D2 pattern used for rehabilitation intervention

\begin{tabular}{|c|c|c|c|}
\hline \multicolumn{2}{|l|}{ D2 flexion Pattern } & \multicolumn{2}{l|}{ D2 extension pattern } \\
\hline Hip & Flexion, Internal rotation, Abduction & Hip & Extension, External rotation, Adduction \\
\hline Foot & Dorsal flexion, Eversion & Foot & Plantar flexion, Inversion \\
\hline Toes & Flexion & Toes & Extension \\
\hline
\end{tabular}




\section{Results}

There were statistically significant differences in the grip power before and after treatment in the experimental group and control group $(P<0.05)$ (Figure 2). In the statistical analysis between groups comparing the size of the effect of treatment, the amount of change in the experimental group treated with combined treatment was significantly larger than that of the control group $(P<0.05)$.

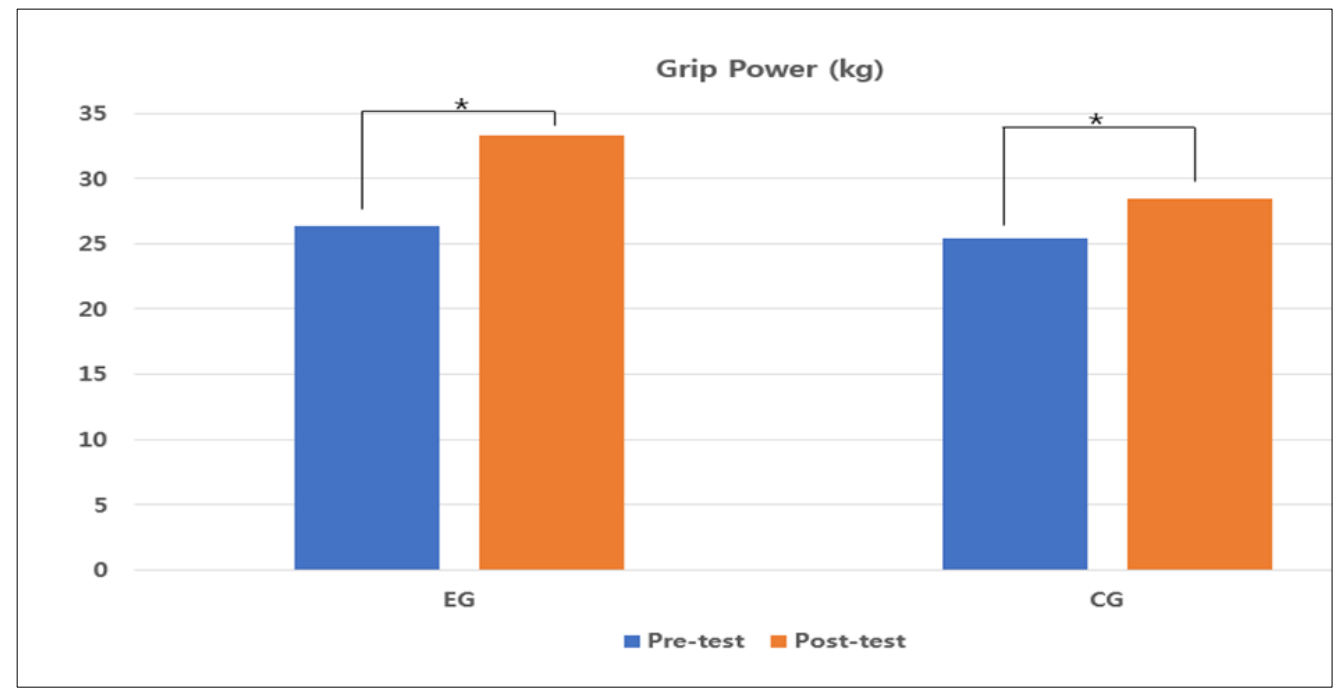

${ }^{*} P<0.05$, EG: Experimental group, CG: Control group

Figure 2 Comparison of grip power between groups

There were statistically significant differences in the muscular strength of the shoulder joint flexor, extensor, adductor and abductor muscles before and after treatment in the experimental group and control group $(P<0.05)$ (Figure 3). In the statistical analysis between groups comparing the effect size of treatment, the amount of change in muscle power around the shoulder was significantly greater in the experimental group treated with combined treatment compared to the control group $(P<0.05)$ (Figure 4$)$.

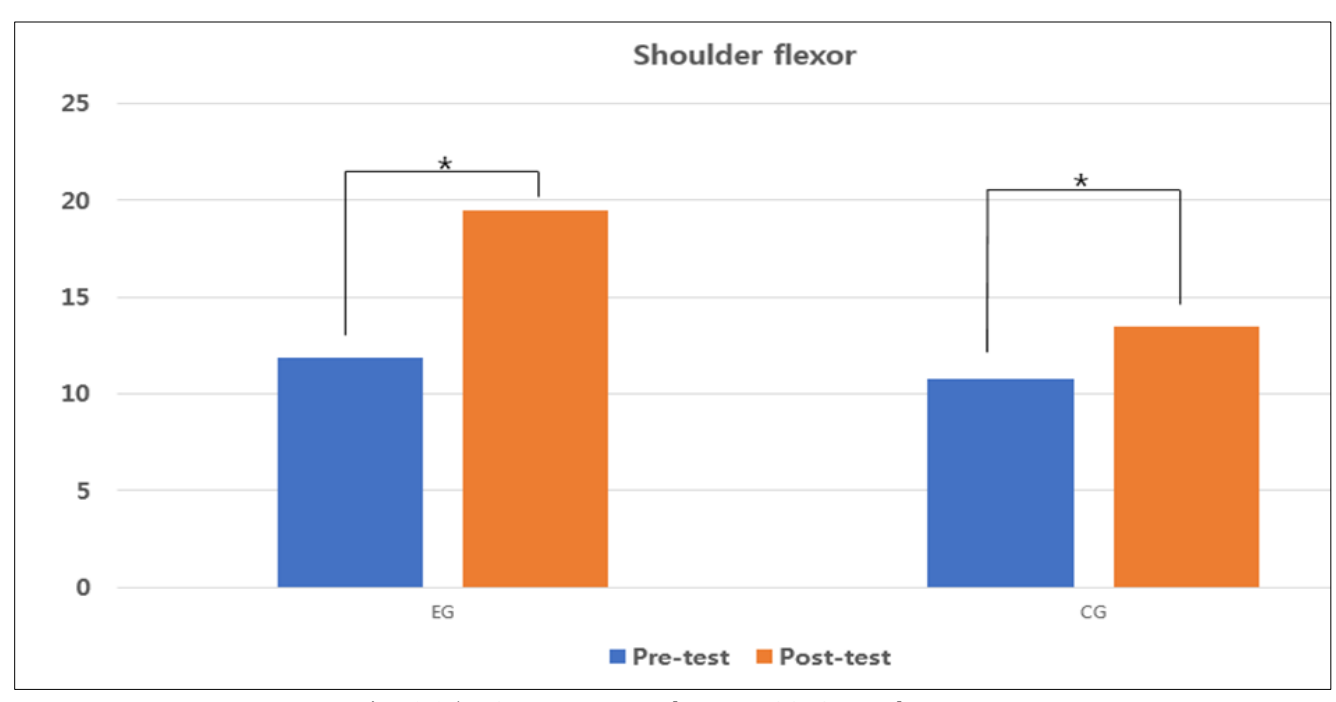

${ }^{*} P<0.05$, EG: Experimental group, CG: Control group

Figure 3 Comparison of flexor strength between groups 


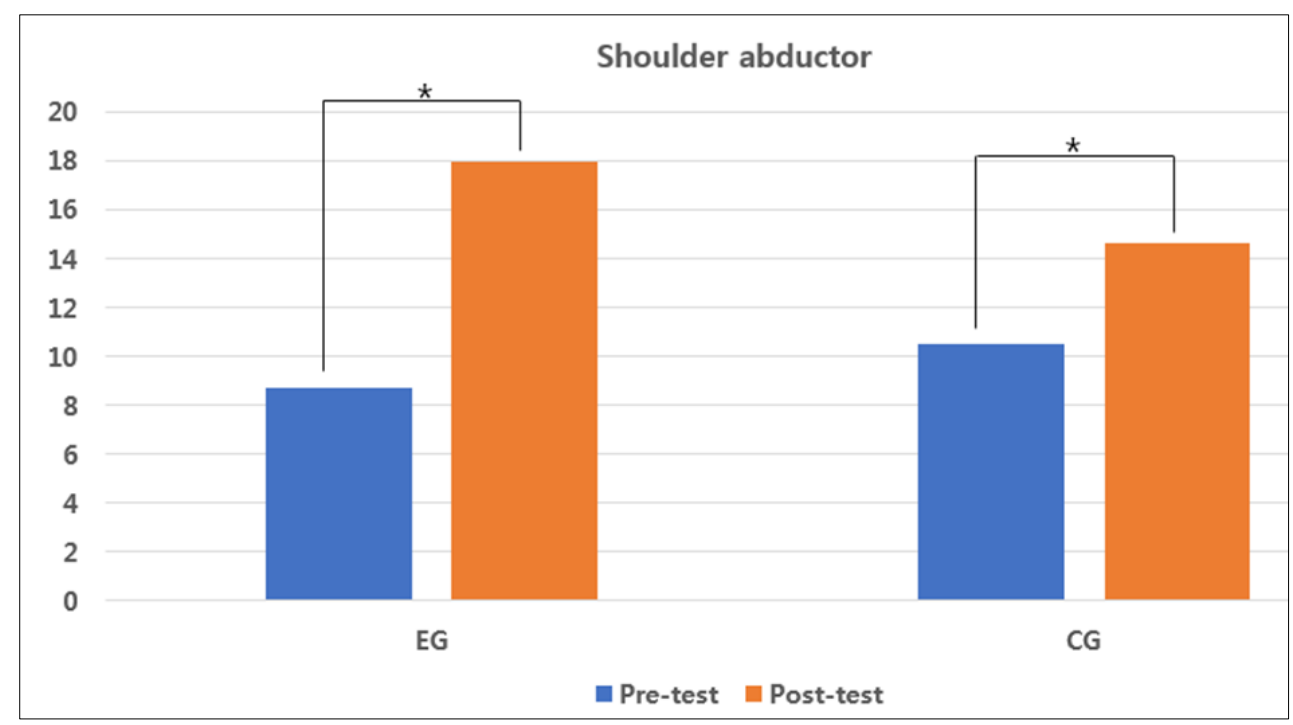

${ }^{*} P<0.05$, EG: Experimental group, CG: Control group

Figure 4 Comparison of abductor strength between groups

\section{Discussion}

Because most upper extremity functions occur with lifting and stretching movements, functional movements against gravity require strength and neuromuscular control [10]. However, muscle weakness in the hand and shoulder joints and inter-joint coordination and timing problems after stroke onset cause abnormal flexion synergy during upper extremity function. This reduces the range of active joint movement, losing the chance to contract, exacerbating muscle weakness over time [11].

In proprioceptive neuromuscular facilitation, movement and postural patterns are altered due to a lack of neuromuscular mechanisms. It can also be used when movement is unnatural, and the main goal of treatment is to restore or reinforce normal patterns of movement or postural response [12,13]. The proprioceptive neuromuscular facilitation technique is currently mainly used for the rehabilitation of patients with the central nervous system in clinical practice, and is used to increase the ability to integrate nerves and muscles through muscle re-education using a diagonal pattern [14]. In addition, the proprioceptive neuromuscular facilitation technique is used not only for the rehabilitation of the nervous system, but also for strengthening and re-educating muscles related to joints among orthopedic diseases [15].

In this study, this proprioceptive neuromuscular facilitation technique was used to increase upper extremity activity in stroke patients with central nervous system damage. As a result of the study, an increase in grip strength and increased activity of muscles around the shoulder joint were observed in the experimental group to which proprioceptive neuromuscular facilitation was applied compared to the control group. This result is thought to be because the proprioceptive neuromuscular facilitation technique, which can stimulate the proprioceptive sensation, stimulates the damaged nerve and increases the activity of the muscles related to the grip force.

Proprioceptive sensation is necessary for correct alignment of the human body and is known to be involved in the timing and activity of precise muscle activity [16]. Many permanent impairments occur after stroke, and impairment of sensory-related proprioceptive sensations can cause secondary damage if not properly treated in the early stages [17, 18]. Elastic taping therapy is often applied in clinical practice to increase the activity of these proprioceptive sensations and to reinforce the muscles of the application site [19].

In this study, taping therapy was applied to the muscles related to the shoulder before treatment for neurorehabilitation. In the study results, the experimental group showed a statistically significant difference in treatment effect compared to the control group, and this result is thought to be due to the maximization of the treatment effect by simultaneously applying two treatment methods that can stimulate proprioceptive sensation. 


\section{Conclusion}

This study was conducted to investigate the effect on the ability of upper extremity activity of stroke patients after central nervous system injury by applying proprioceptive neuromuscular facilitation and taping therapy that can increase proprioceptive sensory activity and applying shock wave therapy that can reduce spasticity. In the results of this study, it was confirmed that the activity of proprioceptive sensation increased according to the overlap of treatment, and the function of stroke patients was improved due to the decrease in spasticity. In other words, for the correct rehabilitation of patients with central nervous system damage, it will be important to combine and apply applicable treatments at an early stage.

\section{Compliance with ethical standards}

\section{Acknowledgments}

This work was supported by the National Foundation of Korea (NRF) grant funded by the Korea government (MSIT) (No-2019R1F1A1057731).

\section{Statement of informed consent}

Informed written consent was obtained from all individual participants included in the study.

\section{References}

[1] Oubre B, Daneault JF, Jung HT, Whritenour K, Miranda JGV, Park J, Ryu T, Kim Y, Lee SI. Estimating Upper-Limb Impairment Level in Stroke Survivors Using Wearable Inertial Sensors and a Minimally-Burdensome Motor Task. Eng Med Biol Soc. 2020; 28(3): 601-611.

[2] McKinnon PJ, Genome Integrity and Disease Prevention in the Nervous System. Genes Dev. 2017; 31(12): 11801194.

[3] Díaz J, Acosta J, González R, Cota J, Sifuentes E, Nebot À. Modeling the Control of the Central Nervous System over the Cardiovascular System Using Support Vector Machines. Comput Biol Med. 2018; 93: 75-83.

[4] Franco J, Imaging and Treatment of Strokes. Radiol Technol. 2018; 89(6): 549-568.

[5] Marciniak C. Poststroke Hypertonicity: Upper Limb Assessment and Treatment. Top Stroke Rehabil. 2011; 18(3): 179-194.

[6] Herman S, Kiely DK, Leveille S, O’Neill E, Cyberey S, Bean JF. Upper and Lower Limb Muscle Power Relationships in Mobility-Limited Older Adults. J Gerontol A Biol Sci Med Sci. 2005; 60(4): 476-480.

[7] Tedla JS, Sangadala DR. Proprioceptive Neuromuscular Facilitation Techniques in Adhesive Capsulitis: A Systematic Review and Meta-Analysis. J Musculoskelet Neuronal Interact. 2019; 19(4): 482-491.

[8] Morreale M, Marchione P, Pili A, Lauta A, Castiglia SF, Spallone A, Pierelli F, Giacomini P. Early versus Delayed Rehabilitation Treatment in Hemiplegic Patients with Ischemic Stroke: Proprioceptive or Cognitive Approach? Eur J Phys Rehabil Med. 2016; 52(1): 81-89.

[9] Williams S, Whatman C, Hume PA, Sheerin K. Kinesio Taping in Treatment and Prevention of Sports Injuries: A Meta-Analysis of the Evidence for Its Effectiveness. Sports Med Auckl NZ. 2012; 42(2): 153-164.

[10] Pérez-Cruzado D, Merchán-Baeza JA, González-Sánchez M, Cuesta-Vargas AI. Systematic Review of Mirror Therapy Compared with Conventional Rehabilitation in Upper Extremity Function in Stroke Survivors. Aust Occup Ther J. 2017; 64(2): 91-112.

[11] Rand D. Proprioception Deficits in Chronic Stroke-Upper Extremity Function and Daily Living. PloS One. 2018; 13(3): e0195043.

[12] Gunning E, Uszynski MK. Effectiveness of the Proprioceptive Neuromuscular Facilitation Method on Gait Parameters in Patients with Stroke: A Systematic Review. Arch Phys Med Rehabil. 2019; 100(5): 980-986.

[13] Ribeiro T, Britto H, Oliveira D, Silva E, Galvão E, Lindquist A. Effects of Treadmill Training with Partial Body Weight Support and the Proprioceptive Neuromuscular Facilitation Method on Hemiparetic Gait: A Randomized Controlled Study. Eur J Phys Rehabil Med. 2013; 49(4): 451-461. 
[14] Klimkiewicz P, Kubsik A, Jankowska A, Woldańska-Okońska M. The effect of standard kinesiotherapy combined with proprioceptive neuromuscular facilitation method and standard kinesiotherapy only on the functional state and muscle tone in patients after ischaemic stroke. Pol Merkur Lek Organ Pol Tow Lek. 2013; 35(209): 268-271.

[15] Sharma V, Kaur J. Effect of Core Strengthening with Pelvic Proprioceptive Neuromuscular Facilitation on Trunk, Balance, Gait, and Function in Chronic Stroke. J Exerc Rehabil. 2017; 13(2): 200-205.

[16] Areeudomwong P, Wongrat W, Neammesri N, Thongsakul T. A Randomized Controlled Trial on the Long-Term Effects of Proprioceptive Neuromuscular Facilitation Training, on Pain-Related Outcomes and Back Muscle Activity, in Patients with Chronic Low Back Pain. Musculoskeletal Care. 2017; 15(3): 218-229.

[17] Bolognini N, Russo C, Edwards DJ. The Sensory Side of Post-Stroke Motor Rehabilitation. Restor Neurol Neurosci. 2016; 34(4): 571-586.

[18] Kessner SS, Bingel U, Thomalla G. Somatosensory Deficits after Stroke: A Scoping Review. Top Stroke Rehabil. 2016; 23(2): 136-146.

[19] Mohammadi R, Abdollahi Khorasgani M, Tabatabaei M, Grampurohit N. Effects of Elastic Therapeutic Taping on Joint Position Sense of the Ankle in Stroke Survivors. Am J Phys Med Rehabil. 2019; 98(9): 830-834. 